\title{
Characterizing the Synchronizability of Small-World Dynamical Networks
}

\author{
Jinhu Lü, Member, IEEE, Xinghuo Yu, Senior Member, IEEE, Guanrong Chen, Fellow, IEEE, and \\ Daizhan Cheng, Senior Member, IEEE
}

\begin{abstract}
Many real-world complex networks display a smallworld feature - a high degree of clustering and a small average distance. We show that the maximum synchronizability of a network is completely determined by its associated feedback system, which has a precise meaning in terms of synchronous communication. We introduce a new concept of synchronizability matrix to characterize the maximum synchronizability of a network. Several new concepts, such as sensitive edge and robust edge, are proposed for analyzing the robustness and fragility of synchronization of a network. Using the knowledge of synchronizability, we can purposefully increase the robustness of the network synchronization and prevent it from attacks. Some applications in small-world networks are also discussed briefly.
\end{abstract}

Index Terms-Associated feedback system, robust edge, sensitive edge, small-world network, synchronizability matrix.

\section{INTRODUCTION}

$\mathbf{M}$ ANY natural and man-made systems, such as nervous systems of living organisms, social systems, the World Wide Web, metabolic systems, food webs, and electrical power grids, can be represented by means of a graph in mathematical terms. In such a graph, the nodes are the single elements of the system, such as neurons in the brain, human beings in a society, a hyperlink in the WWW, etc, while the edges are connections preparing measures of the interactions among the individual elements [1], [2]. These graphs are called complex networks. Over the last decade, complex networks have been intensively studied in many fields, such as social, biological, mathematical, and engineering sciences.

To well understand the complex dynamical behaviors of many natural systems, we need to study the topological structures of the underlying networks. In fact, the properties of a complex network are mainly determined by its topological structure - connections between nodes. At one extreme are the regular networks, such as chains, lattices, grids, and fully connected graphs, while at the other extreme are the random networks

Manuscript received March 27, 2003; revised September 1, 2003. This work was supported in part by the Hong Kong Research Grants Council under Grant CityU 1004/02E, in part by the K. C. Wong Education Foundation, Hong Kong, and in part by the National Natural Science Foundation, China under Grant 60304017. This paper was recommended by Associate Editor R. Dogaru.

J. Lü and D. Cheng are with the Institute of Systems Science, Academy of Mathematics and System Science, Chinese Academy of Sciences, Beijing 100080, China (e-mail: 1vjinhu@ mail.amss.ac.cn; dcheng@ iss03.iss.ac.cn).

$\mathrm{X}$. Yu is with the School of Electrical and Computer Engineering, Faculty of Engineering, RMIT University, Melbourne VIC 3001, Australia (e-mail: x.yu@ rmit.edu.au).

G. Chen is with the Department of Electronic Engineering, City University of Hong Kong, Hong Kong (e-mail: gchen@ee.cityu.edu.hk).

Digital Object Identifier 10.1109/TCSI.2004.823672 where the nodes are connected randomly. Regular networks are clustered but do not exhibit the small-world effect [3]-[5]. However, random networks display the small-world effect but do not show clustering. To bridge the gap between these two extreme cases, Watts and Strogatz introduced an interesting concept of small-world network [4]. In general, small-world networks have intermediate connectivity properties but exhibit a high degree of clustering as in the regular networks and a small average distance between two nodes as in the random networks. Small-world networks have an ordered local structure but are random on a global scale. Small-world networks are characterized by two quantities: a high clustering coefficient $C$ like regular lattices and a short average path length $L$ like random graphs. $C$ represents the degree of local order and is defined to be the probability that two nodes connected to a common node are also connected to each other. $L$, which measures the efficiency of information propagation between nodes, is defined to be the average number of links in the shortest path between a pair of nodes in the network [6]. It has been demonstrated that many real-world networks, such as social networks, metabolic networks, communication networks, power grids, and collaboration graphs of film actors, display small-world behaviors. Many numerical and analytic results for small-world networks have been obtained in several directions, including the empirical work for determining the exact structure of a real-world network and the theoretical work for studying the properties of various network models. It has been found that small-world networks have many applications in various fields, such as social studies, scientific collaboration, the Internet, telecommunication, business, and life sciences [7].

Collective motions of complex networks have recently been the subject of considerable interest within the science and technology communities. Especially, one of the interesting and significant phenomena in complex dynamical networks is the synchronization of all dynamical nodes in a network [8]-[11], [14]-[16]. In fact, synchronization is a kind of basic motions in nature. The synchronization of coupled oscillators can well explain many natural phenomena [8], [12]-[14]. Moreover, many real-world problems have close relationships with network synchronization, such as the synchronous phenomena on the Internet, and the synchronous transfer of digital or analog signals in the communication networks. Recently, synchronization in networks of coupled chaotic systems has received a great deal of attention [8]-[26]. Most of the existing works have focused on completely regular networks, such as the continuous-time cellular neural network (CNN) and the discrete-time coupled map lattice (CML) [14], while some studies 


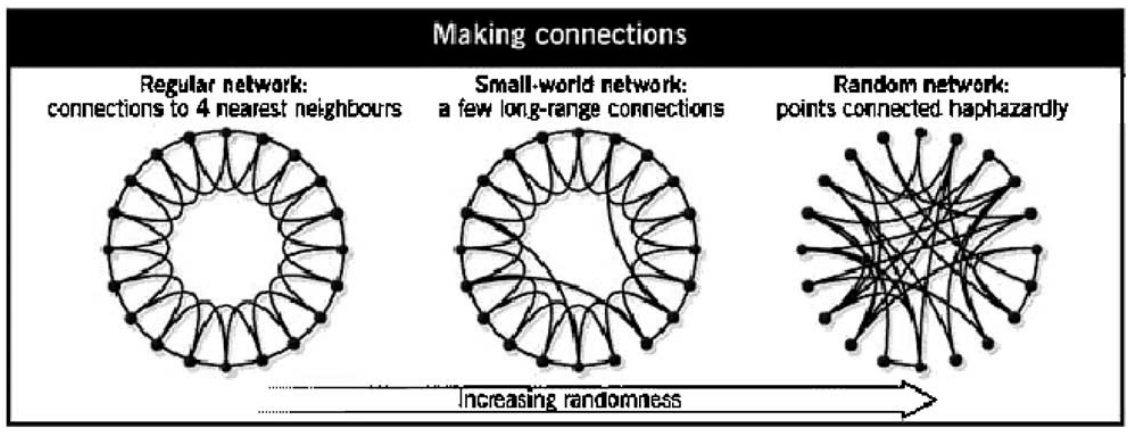

Fig. 1. Formation of a small-world network [7].

address the synchronization of randomly coupled networks [15]. However, many real-world networks, such as the WWW, food webs, and metabolic networks are neither completely regular nor completely random. Knowing this, very recently, Wang and Chen have further investigated the synchronization of small-world and scale-free networks [8], [9].

Many complex networks display a surprising degree of robustness against disturbances and network errors. For example, simple organisms may grow, produce, and persist despite drastic pharmaceutical or environmental interventions, an error tolerance attributed to the robustness of the underlying metabolic network [27]. Complex communication networks also display a surprising degree of robustness: although key components regularly malfunction, local failures rarely lead to the global loss of network information-carrying ability.

Although there are many papers which discuss the network synchronization problem [8]-[10], [15], [23]-[26], there are few results reported in the literature on how to rigorously characterize the network synchronizability. In fact, so far, we are still not able to answer the basic question "What is the maximum synchronizability of a complex network?" Most of the existing works were based on numerical or statistical results [23], lack of rigorous theoretical analysis. In this paper, we show that maximum synchronizability of a network is completely determined by its associated feedback system, which has a precise meaning in terms of synchronous communication, based on some rigorous mathematical methods. We introduce a new concept of synchronizability matrix to characterize the robustness of synchronization of a network. At the same time, the concepts of sensitive edge and robust edge of a network are defined based on the synchronizability matrix. Using the knowledge of synchronizability, we can purposefully increase the maximum synchronizability to, for example, prevent the network from attacks. To that end, some applications in small-world networks are discussed briefly.

This paper is organized as follows: The Watts and Strogatz (WS) model is first reviewed, and a general dynamical network model and its synchronization theorems are then proposed in Section II. In Section III, the concepts of associated feedback system, synchronizability matrix, robust edge, and sensitive edge of a network are introduced to characterize the synchronizability of a dynamical network. An example is analyzed, and some applications are discussed in Section IV. Conclusions are given in Section V.

\section{PRELIMINARIES}

\section{A. Small-World Models}

In 1998, Watts and Strogatz introduced a single-parameter small-world network model that bridges the gap between a regular network and a random graph [4]. The original WS model is described as follows.

1) Start with order: Start with a nearest-neighbor coupled ring lattice with $N$ nodes, in which each node $i$ is connected to its $K$ neighboring nodes $i \pm 1, i \pm 2, \ldots, i \pm K / 2$, where $K$ is an even integer. Assume that $N \gg K \gg$ $\ln (N) \gg 1$, which guarantees that the network is connected but sparse at all times.

2) Randomize: Randomly rewire each link of the network with probability $p$ such that self-connections and duplicated links are excluded. Rewiring in this sense means transferring one end of the connection to a randomly chosen node. This process introduces $(p N K / 2)$ long-range links, which connect some nodes that otherwise would not have direct connections. One can thus closely monitor the transition between order $(p=0)$ and randomness $(p=1)$ by adjusting $p$.

Fig. 1 shows that a small-world network lies along a continuum of network models between the two extreme networks: regular and random ones. Lately, Newman and Watts improved the original WS model [5]. Most of the recent works on smallworld models were performed using the Newman and Watts (NW) model. The NW model, instead of rewiring links between nodes, extra links called shortcuts are added between pairs of nodes chosen at random, but no links are removed from the existing network. Obviously, the NW model reduces to the originally nearest-neighbor coupled network for $p=0$; while it becomes a globally coupled network for $p=1$. Moreover, the NW model is equivalent to the WS model for sufficiently small $p$ and large $N$. The WS and NW models show a transition with an increasing number of nodes from a "large-world" regime in which the average distance between two nodes increases linearly with the system size, to a "small-world" one in which it increases only logarithmically.

\section{B. General Dynamical Network Model}

Recently, Wang and Chen have proposed a uniformly dynamical network model [9], which has the same coupling strength 
for all edges and the inner coupling matrix is a constant $0-1$ diagonal matrix. However, most real-world complex networks have different coupling strengths for different edges, and their inner coupling matrix is required to not to be a diagonal matrix. To better characterize the real-world complex networks, a general dynamical network model is introduced here

$$
\dot{\mathbf{x}}_{i}(t)=\mathbf{f}\left(\mathbf{x}_{i}(t)\right)+\sum_{j=1}^{N} c_{i j} \mathbf{A} \mathbf{x}_{j}(t), \quad i=1,2, \ldots, N
$$

where $\mathbf{x}_{i}(t)=\left(x_{i 1}(t), x_{i 2}(t), \ldots, x_{i n}(t)\right)^{T} \in \mathbf{R}^{n}$ are the state variables of node $i, \mathbf{A}=\left(a_{i j}\right)_{n \times n} \in \mathbf{R}^{n \times n}$ is a constant inner coupling matrix between nodes, and $\mathbf{C}=\left(c_{i j}\right)_{N \times N}$ is the coupling configuration matrix of the network, where $c_{i j}$ is defined as follows: If there is a connection from node $i$ to node $j(j \neq i)$, then, the coupling strength $c_{i j} \neq 0$; otherwise, $c_{i j}=0(j \neq i)$, and the diagonal elements of $\mathbf{C}$ are defined by

$$
c_{i i}=-\sum_{\substack{j=1 \\ j \neq i}}^{N} c_{i j}, \quad i=1,2, \ldots, N .
$$

Obviously, the uniform network modeled in [9] is a special case of network (1), where $\mathbf{C}$ is a $0-1$ symmetric matrix and $\mathbf{A}$ is a 0-1 diagonal matrix. Since real-world complex networks may be directed networks, such as the WWW, whose coupling configuration matrix $\mathbf{C}$ is not symmetric, here it is not assumed that $\mathbf{C}$ is symmetric and its off-diagonal elements are nonnegative.

Note that matrix $\mathbf{C}$ has some interesting properties. According to [16, Lemma 2], the real parts of all eigenvalues of matrix $\mathbf{C}$ are less than or equal to 0 , and all eigenvalues with zero real part are the real eigenvalue 0. Hereafter, suppose that $\mathbf{C}$ is an irreducible matrix; therefore, 0 is an eigenvalue of multiplicity 1 [16]. If all the eigenvalues of $\mathbf{C}$ are real numbers satisfying $\lambda_{1} \geq \lambda_{2} \geq \cdots \geq \lambda_{N}$, then

$$
\lambda_{1}=0 \quad \text { and } \quad 0>\lambda_{2} \geq \cdots \geq \lambda_{N} .
$$

\section{Several Lemmas}

At first, a rigorous mathematical definition is introduced for the concept of network synchronization.

Definition 1: Let $\mathbf{x}_{i}\left(t ; t_{0}, \mathbf{x}_{1}^{0}, \ldots, \mathbf{x}_{N}^{0}\right)(i=1,2, \ldots, N)$ be a solution for the dynamical network

$$
\begin{array}{r}
\dot{\mathbf{x}}_{i}(t)=\mathbf{f}\left(\mathbf{x}_{i}(t)\right)+\mathbf{g}_{i}\left(\mathbf{x}_{1}(t), \mathbf{x}_{2}(t), \ldots, \mathbf{x}_{N}(t)\right), \\
i=1,2, \ldots, N
\end{array}
$$

where $\mathbf{f}: D \rightarrow \mathbf{R}^{n}$ and $\mathbf{g}_{i}: D \times \cdots \times D \rightarrow \mathbf{R}^{n}(i=$ $1,2, \ldots, N)$ are continuously differentiable, $D \subseteq \mathbf{R}^{n}$. If there is a nonempty open subset $D^{0}\left(t_{0}\right) \subseteq D$, with $\mathbf{x}_{i}^{0} \in D^{0}\left(t_{0}\right)(i=$ $1,2, \ldots, N)$, such that $\mathbf{x}_{i}\left(t ; t_{0}, \mathbf{x}_{1}^{0}, \ldots, \mathbf{x}_{N}^{0}\right) \in D^{0}\left(t_{0}\right)$ for all $t \geq t_{0}, i=1,2, \ldots, N$, and

$\lim _{t \rightarrow \infty}\left\|\mathbf{x}_{i}\left(t ; t_{0}, \mathbf{x}_{1}^{0}, \ldots, \mathbf{x}_{N}^{0}\right)-\mathbf{x}_{j}\left(t ; t_{0}, \mathbf{x}_{1}^{0}, \ldots, \mathbf{x}_{N}^{0}\right)\right\|_{2}=\mathbf{0}$,

$$
\text { for } 1 \leq i, j \leq N
$$

then, the dynamical network (4) is said to realize synchronization and $D^{0}\left(t_{0}\right) \times \cdots \times D^{0}\left(t_{0}\right)$ is called the region of synchrony for the dynamical network (4).
Note that the diffusively coupled condition (2) of network (1) ensures that the synchronous solution $\mathbf{x}_{1}\left(t ; t_{0}, \mathbf{x}_{1}^{0}, \ldots, \mathbf{x}_{N}^{0}\right)=$ $\mathbf{x}_{2}\left(t ; t_{0}, \mathbf{x}_{1}^{0}, \ldots, \mathbf{x}_{N}^{0}\right)=\cdots=\mathbf{x}_{N}\left(t ; t_{0}, \mathbf{x}_{1}^{0}, \cdots, \mathbf{x}_{N}^{0}\right)\left(\mathbf{x}_{1}^{0}=\right.$ $\left.\cdots=\mathbf{x}_{N}^{0} \in D\right)$ be a solution of an individual node described by $\dot{\mathbf{x}}(t)=\mathbf{f}(\mathbf{x}(t))$, denoted as $\mathbf{s}(t)$, namely

$$
\dot{\mathbf{s}}(t)=f(\mathbf{s}(t)) \text {. }
$$

Obviously, synchronization in network (1) corresponds to the motion in the invariant manifold: $\mathbf{x}_{1}(t)=\mathbf{x}_{2}(t)=\cdots=$ $\mathbf{x}_{N}(t)$. Here, $\mathbf{s}(t)$ can be either an equilibrium point, or a periodic orbit, or an orbit of a chaotic attractor.

It is very important to point out that since a chaotic attractor is an attracting invariant set, the stability of the chaotic synchronous state $\mathbf{x}_{1}(t)=\mathbf{x}_{2}(t)=\cdots=\mathbf{x}_{N}(t)$ is equivalent to the stability of the zero transverse errors of the synchronous manifold for the dynamical network (1) [10]. However, it is quite different from the nonchaotic case. Since it is not assumed the stability of $\mathbf{s}(t)$, then the stability of the synchronous solution $\left(\mathbf{s}^{T}(t), \mathbf{s}^{T}(t), \ldots, \mathbf{s}^{T}(t)\right)^{T}$ of network (1) is equivalent to the stability of the error vector $\left(\eta_{1}^{T}(t), \eta_{2}^{T}(t), \ldots, \eta_{N}^{T}(t)\right)$ about its zero solution, where $\eta_{i}(t)=\mathbf{x}_{i}(t)-\mathbf{s}(t)(i=1,2, \ldots, N)$.

In the following, we consider two cases: 1) chaos synchronization of network (1) and 2) nonchaotic synchronization of network (1).

1) Case 1): Chaos Synchronization: Suppose that $\dot{\mathbf{x}}(t)=$ $\mathbf{f}(\mathbf{x}(t))$ is a chaotic system and $\mathbf{s}(t)$ is one of the orbits of its chaotic attractor. Denote

$$
\begin{aligned}
\dot{\eta}_{i}(t)=\mathbf{f}\left(\mathbf{s}(t)+\eta_{i}(t)\right)- & \mathbf{f}(\mathbf{s}(t)) \\
& +\sum_{j=2}^{N} c_{i j} \mathbf{A} \eta_{i}(t), \quad 2 \leq i \leq N
\end{aligned}
$$

where $\mathbf{x}_{1}(t)=\mathbf{s}(t)$ is the reference direction of synchronous manifold [10], $\eta_{i}(t)=\mathbf{x}_{i}(t)-\mathbf{s}(t)(2 \leq i \leq N)$, and $\bar{\eta}(t)=$ $\left(\eta_{2}^{T}(t), \eta_{3}^{T}(t), \ldots, \eta_{N}^{T}(t)\right)^{T} \in \mathbf{R}^{n(N-1)}$. Then, (7) can be written as

$$
\dot{\bar{\eta}}(t)=\mathbf{F}(t, \overline{\eta(} t))
$$

Denote by $\mu(\mathbf{A})$ the maximum eigenvalue of matrix $1 / 2\left(\mathbf{A}^{T}+\right.$ A).

Hypothesis 1: (H1) Assume that $\mathbf{F}: \Omega \rightarrow \mathbf{R}^{n(N-1)}$ is continuously differentiable, $\Omega=\left\{\mathbf{x} \in \mathbf{R}^{n(N-1)}|\| \mathbf{x}|_{2}<r\right\}$, the Jacobian matrix $D \mathbf{F}(t, \mathbf{x}(t))$ is bounded and Lipschitz on $\Omega$, uniformly in $t$, and the coupling configuration matrix $\mathbf{C}$ can be diagonalized.

Lemma 1: Suppose that (H1) holds. The chaotic synchronous state $\mathbf{x}_{1}(t)=\mathbf{x}_{2}(t)=\cdots=\mathbf{x}_{N}(t)=\mathbf{s}(t)$ of dynamical network (1) is exponentially stable if and only if the linear time-varying systems

$$
\dot{\mathbf{w}}(t)=\left[D \mathbf{f}(\mathbf{s}(t))+\lambda_{k} \mathbf{A}\right] \mathbf{w}(t) \quad k=2, \ldots, N
$$

are exponentially stable about their zero solutions, where $\lambda_{k}(2 \leq k \leq N)$ are the nonzero eigenvalues of matrix $\mathbf{C}$.

The proof of Lemma 1 is similar to that of Lemma 1 in [9]. Note that Lemma 1 is a sufficient and necessary condition for chaos synchronization. Since the Jacobian matrix $D \mathbf{F}(t, \mathbf{x}(t))$ is bounded and Lipschitz on $\Omega$, uniformly in $t$, according to the Lyapunov converse theorem [28], the origin is an exponentially 
stable equilibrium point for the nonlinear system (8) if and only if it is an exponentially stable equilibrium point for the linear time-varying system

$$
\dot{\bar{\eta}}(t)=D \mathbf{F}(t, \mathbf{0}) \bar{\eta}(t) .
$$

Moreover, the origin is an exponentially stable equilibrium point for the linear system (10) if and only if $\bar{\eta}(t) \rightarrow \mathbf{0}$ exponentially as $t \rightarrow+\infty$. Since $\eta_{1}(t)=\mathbf{x}_{1}(t)-\mathbf{s}(t) \equiv \mathbf{0}$, one has $\bar{\eta}(t) \rightarrow \mathbf{0}$ exponentially as $t \rightarrow+\infty$ if and only if $\eta(t) \rightarrow \mathbf{0}$ exponentially as $t \rightarrow+\infty$, where $\eta(t)=\left(\eta_{1}(t), \eta_{2}(t), \cdots, \eta_{N}(t)\right) \in \mathbf{R}^{n \times N}$. The rest of the proof is similar to the proof of [9, Lemma 1] and, therefore, is omitted.

Lemma 2: Suppose that (H1) holds. Assume that all eigenvalues of $\mathbf{C}$ are real numbers satisfying $\lambda_{1} \geq \lambda_{2} \geq \cdots \geq \lambda_{N}$. If $\left(\mathbf{A}+\mathbf{A}^{T}\right)$ is a positive semi-definite matrix, and $\mu[D \mathbf{f}(\mathbf{s}(t))+$ $\left.\lambda_{2} \mathbf{A}\right] \leq a<0$ for all $t \geq t_{0}$, then, the chaotic synchronous state $\mathbf{x}_{1}(t)=\mathbf{x}_{2}(t)=\cdots=\mathbf{x}_{N}(t)=\mathbf{s}(t)$ of dynamical network (1) is exponentially stable.

Proof: Since all eigenvalues of $\mathbf{C}$ are real numbers, from (3), $\lambda_{1}=0$ and $0>\lambda_{2} \geq \cdots \geq \lambda_{N}$. Consider the linear timevarying systems (9). Since $\left(\mathbf{A}+\mathbf{A}^{T}\right)$ is a positive semi-definite matrix, one has

$$
\begin{aligned}
\frac{1}{2} & \frac{d\|\mathbf{x}(t)\|^{2}}{d t} \\
= & \mathbf{x}^{T}(t)\left(\frac{\left(D \mathbf{f}(\mathbf{s}(t))+\lambda_{2} \mathbf{A}\right)^{T}+\left(D \mathbf{f}(\mathbf{s}(t))+\lambda_{2} \mathbf{A}\right)}{2}\right) \\
\quad & \times \mathbf{x}(t)\left(\lambda_{k}-\lambda_{2}\right) \mathbf{x}^{T}(t)\left(\frac{\mathbf{A}^{T}+\mathbf{A}}{2}\right) \mathbf{x}(t) \\
\leq & \mathbf{x}^{T}(t)\left(\frac{\left(D \mathbf{f}(\mathbf{s}(t))+\lambda_{2} \mathbf{A}\right)^{T}+\left(D \mathbf{f}(\mathbf{s}(t))+\lambda_{2} \mathbf{A}\right)}{2}\right) \mathbf{x}(t)
\end{aligned}
$$

for $k=2,3, \ldots, N$. Since $\mu\left[D \mathbf{f}(\mathbf{s}(t))+\lambda_{2} \mathbf{A}\right] \leq a<0$ for all $t \geq t_{0}$, one has $\|\mathbf{x}(t)\| \leq\left\|\mathbf{x}\left(t_{0}\right)\right\| e^{a t}$. That is, all linear time-varying systems (9) are exponentially stable about their zero solutions. From Lemma 1, the chaotic synchronous state of dynamical network (1) is exponentially stable. The proof is thus completed.

2) Case 2): Nonchaotic Synchronization: Let $\mathbf{x}(t)=\mathbf{s}(t)$ be an exponentially stable solution of the individual node $\dot{\mathbf{x}}(t)=\mathbf{f}(\mathbf{x}(t))$. Denote

$$
\begin{array}{r}
\dot{\eta}_{i}(t)=\mathbf{f}\left(\mathbf{s}(t)+\eta_{i}(t)\right)-\mathbf{f}(\mathbf{s}(t))+\sum_{j=1}^{N} c_{i j} \mathbf{A} \eta_{i}(t), \\
1 \leq i \leq N
\end{array}
$$

where $\eta_{i}(t)=\mathbf{x}_{i}(t)-\mathbf{s}(t)(1 \leq i \leq N), \hat{\eta}(t)=$ $\left(\eta_{1}^{T}(t), \eta_{2}^{T}(t), \ldots, \eta_{N}^{T}(t)\right)^{T} \in \mathbf{R}^{n N}$. Then, (11) can be written as

$$
\dot{\hat{\eta}}(t)=\hat{\mathbf{F}}(t, \hat{\eta}) .
$$

Hypothesis 2: (H2) Assume that $\hat{\mathbf{F}}: \hat{\Omega} \rightarrow \mathbf{R}^{n N}$ is continuously differentiable, $\hat{\Omega}=\left\{\mathbf{x} \in \mathbf{R}^{n N} \mid\|\mathbf{x}\|_{2}<r\right\}$, the Jacobian matrix $D \hat{\mathbf{F}}(t, \mathbf{x}(t))$ is bounded and Lipschitz on $\Omega$, uniformly in $t$, and the coupling configuration matrix $\mathbf{C}$ can be diagonalized.

Lemma 3: Suppose that (H2) holds. The synchronous solution $\mathbf{S}(t)=\left(\mathbf{s}^{T}(t), \mathbf{s}^{T}(t), \ldots, \mathbf{s}^{T}(t)\right)^{T}$ of dynamical network
(1) is exponentially stable if and only if the linear time-varying systems

$$
\dot{\mathbf{w}}(t)=\left[D \mathbf{f}(\mathbf{s}(t))+\lambda_{k} \mathbf{A}\right] \mathbf{w}(t), \quad k=2, \ldots, N
$$

are exponentially stable about their zero solutions, where $\lambda_{k}(2 \leq k \leq N)$ are the nonzero eigenvalues of matrix $\mathbf{C}$.

The proof of Lemma 3 is also similar to that of Lemma 1 in [9]. Here, Lemma 3 is a sufficient and necessary condition. Since the Jacobian matrix $D \hat{\mathbf{F}}(t, \mathbf{x}(t))$ is bounded and Lipschitz on $\hat{\Omega}$, uniformly in $t$, from the Lyapunov converse theorem [28], the origin is an exponentially stable equilibrium point for the nonlinear system (12) if and only if it is an exponentially stable equilibrium point for the linear system

$$
\dot{\hat{\eta}}(t)=D \hat{\mathbf{F}}(t, \mathbf{0}) \hat{\eta}(t) .
$$

Moreover, the origin is an exponentially stable equilibrium point for the linear system (14) if and only if $\hat{\eta}(t) \rightarrow \mathbf{0}$ exponentially as $t \rightarrow+\infty$, and $\hat{\eta}(t) \rightarrow \mathbf{0}$ exponentially as $t \rightarrow+\infty$ if and only if $\eta(t) \rightarrow \mathbf{0}$ exponentially as $t \rightarrow+\infty$, where $\eta(t)=$ $\left(\eta_{1}(t), \eta_{2}(t), \ldots, \eta_{N}(t)\right) \in \mathbf{R}^{n \times N}$. The rest of proof is similar to the proof of [9, Lemma 1] and so, is omitted.

Lemma 4: Suppose that (H2) holds. Assume that all eigenvalues of $\mathbf{C}$ are real numbers satisfying $\lambda_{1} \geq \lambda_{2} \geq \cdots \geq \lambda_{N}$. If $\left(\mathbf{A}+\mathbf{A}^{T}\right)$ is a positive semi-definite matrix, and $\mu[D \mathbf{f}(\mathbf{s}(t))] \leq$ $a<0$ for all $t \geq t_{0}$, then, the synchronous solution $\mathbf{S}(t)$ of dynamical network (1) is exponentially stable.

The proof of Lemma 4 is similar to that of Lemma 2 and is omitted here.

It is noticed that Lemmas 1 and 3 have the same result under the conditions of (H1) and (H2), respectively. It means that, whether $\mathbf{s}(t)$ is an orbit of a chaotic attractor or an exponentially stable solution, network (1) achieves synchronization under the same conditions: Systems (9) or (13), respectively, are exponentially stable about their zero solutions.

\section{CHARACTERIZATION OF SYNCHRONIZABILITY}

\section{A. Maximum Synchronizability of Dynamical Networks}

Network synchronizability is an important property of complex dynamical networks. Networks with different topological structures have different degrees of network synchronizability. It has been demonstrated that, for any given coupling strength, if the number of nodes is sufficiently large, then the smallworld dynamical network will synchronize, even if the original nearest-neighbor coupled network cannot realize synchronization under the same condition [8]. However, how to characterize the synchronizability of a network is an open problem. In the following, a new concept—associated feedback system - is introduced for characterizing synchronizability of network (1).

Definition 2: The self-feedback nonlinear system

$$
\dot{\mathbf{x}}(t)=\mathbf{f}(\mathbf{x}(t))+d \mathbf{A}(\mathbf{x}(t)-\mathbf{s}(t))
$$

where $\mathbf{x}=\left(\mathbf{x}_{1}, \mathbf{x}_{2}, \ldots, \mathbf{x}_{n}\right)^{T} \in \mathbf{R}^{n}$ and $d$ is a constant, is called the associated feedback system of the dynamical network (1).

Letting $\mathbf{x}(t)=\mathbf{y}(t)+\mathbf{s}(t)$, and substituting it into (15), yields

$$
\dot{\mathbf{y}}(t)=\mathbf{f}(\mathbf{y}(t)+\mathbf{s}(t))-\mathbf{f}(\mathbf{s}(t))+d \mathbf{A y}(t)
$$


and its corresponding linear system is

$$
\dot{\mathbf{y}}(t)=[D \mathbf{f}(\mathbf{s}(t))+d \mathbf{A}] \mathbf{y}(t) .
$$

For the given dynamical network (1), one can obtain the exponentially stable region, denoted as $\Gamma$, of the solution $\mathbf{s}(t)$ of the associated feedback system (15) [or the zero solution of system (16)] in terms of feedback parameter $d$.

Theorem 1: Let $\mathbf{s}(t)$ be an orbit of a chaotic attractor of the given chaotic system $\dot{\mathbf{x}}(t)=\mathbf{f}(\mathbf{x}(t))$. Suppose that (H1) holds. The chaotic synchronous state $\mathbf{x}_{1}(t)=\mathbf{x}_{2}(t)=\cdots=\mathbf{x}_{N}(t)=$ $\mathbf{s}(t)$ of dynamical network (1) is exponentially stable if and only if the eigenvalues $\lambda_{i} \in \Gamma, i=2,3, \ldots N$.

Proof: From (H1), the Jacobian matrix $D \mathbf{f}(\mathbf{s}(t))$ is bounded and Lipschitz on $\bar{\Omega}=\left\{\mathbf{y} \in \mathbf{R}^{n}\|\| \mathbf{y} \|_{2}<r\right\}$, uniformly in $t$. According to the Lyapunov converse theorem [28], the origin is an exponentially stable solution for the nonlinear system (16) if and only if it is an exponentially stable equilibrium point for the linear time-varying system (17). Therefore, $\Gamma$ is also the exponentially stable region of the zero solution of the linear time-varying system (17) with respect to feedback parameter $d$. From Lemma 1, the chaotic synchronous state $\mathbf{x}_{1}(t)=\mathbf{x}_{2}(t)=\cdots=\mathbf{x}_{N}(t)=\mathbf{s}(t)$ of dynamical network (1) is exponentially stable if and only if all the eigenvalues $\lambda_{i} \in \Gamma, i=2,3, \ldots N$. The proof is thus completed.

Theorem 2: Let $\mathbf{x}(t)=\mathbf{s}(t)$ be an exponentially stable solution of the individual node $\dot{\mathbf{x}}(t)=\mathbf{f}(\mathbf{x}(t))$. Suppose that (H2) holds. The synchronous solution $\mathbf{S}(t)=\left(\mathbf{s}^{T}(t), \mathbf{s}^{T}(t), \ldots, \mathbf{s}^{T}(t)\right)^{T}$ of dynamical network (1) is exponentially stable if and only if all the eigenvalues $\lambda_{i} \in \Gamma, i=2,3, \ldots N$.

Proof: According to (H2), the Jacobian matrix $D \mathbf{f}(\mathbf{s}(t))$ is bounded and Lipschitz on $\bar{\Omega}=\left\{\mathbf{y} \in \mathbf{R}^{n} \mid\|\mathbf{y}\|_{2}<r\right\}$, uniformly in $t$. From the Lyapunov converse theorem [28], the origin is an exponentially stable solution for the nonlinear system (16) if and only if it is an exponentially stable equilibrium point for the linear time-varying system (17). Therefore, $\Gamma$ is also the exponentially stable region of the zero solution of the linear time-varying system (17) in terms of feedback parameter $d$. From Lemma 3, the synchronous solution $\mathbf{S}(t)$ of dynamical network (1) is exponentially stable if and only if all the eigenvalues $\lambda_{i} \in \Gamma, i=2,3, \ldots N$. This thus completes the proof.

Remarks:

1) Theorems 1 and 2 give sufficient and necessary conditions for the exponential stability of the synchronous solution of network (1). It is noticed that the stable region $\Gamma$ is completely determined by the individual node $\dot{\mathbf{x}}(t)=\mathbf{f}(x)$ and the inner coupled matrix $\mathbf{A}$ of network (1) and that the eigenvalues of the coupled configuration matrix $\mathbf{C}$ determine the stability of synchronous solution of network (1).

2) $\Gamma$ is the maximum region of the eigenvalues of the coupled configuration matrix $\mathbf{C}$. Note that Theorems 1 and 2 give the same result under different conditions of (H1) and $(\mathrm{H} 2)$.

Note that for a given individual node $\dot{\mathbf{x}}(t)=\mathbf{f}(\mathbf{x}(t))$, the network synchronizability is completely determined by the inner coupling matrix $\mathbf{A}$ and the coupling configuration matrix $\mathbf{C}$. It is noticed that the unique hypothesis for matrix $\mathbf{C}$ is the diagonalized condition, which is a rather general condition for complex networks. However, under the condition of positive semi-definite for $\left(\mathbf{A}+\mathbf{A}^{T}\right)$, we can derive some very good results as shown in Lemma 4. Moreover, $\left(\mathbf{A}+\mathbf{A}^{T}\right)$ is a positive semi-definite matrix for many real-world small-world networks.

Definition 3: The ability that the structure of network (1) can ensure network (1) achieve synchronization is called the network synchronizability. The maximum possible set

$$
\{(\mathbf{A}, \mathbf{C}) \mid \text { network (1) realizes synchronization }\}
$$

is called the maximum synchronizability set, which characterizes the maximum synchronizability of network (1).

Obviously, for a given individual node $\dot{\mathbf{x}}(t)=\mathbf{f}(\mathbf{x}(t))$ and a inner coupling matrix $\mathbf{A}$, the maximum synchronizability set of network (1) is completely determined by its associated feedback system (15). In fact, the maximum synchronizability set of network (1) is

$$
\left\{\mathbf{C} \mid \lambda_{i} \in \Gamma \text { for } i=2,3, \cdots, N\right\}
$$

where $\lambda_{i}(i=2,3, \ldots, N)$ are the nonzero eigenvalues of $\mathbf{C}$. Consider the following unidirectional coupled system:

$$
\left\{\begin{array}{l}
\dot{\mathbf{s}}(t)=\mathbf{f}(\mathbf{s}(t)) \\
\dot{\mathbf{x}}(t)=\mathbf{f}(\mathbf{x}(t))+d \mathbf{A}(\mathbf{x}(t)-\mathbf{s}(t))
\end{array}\right.
$$

where $\mathbf{A}$ is a constant coupled matrix, and $d$ is a coupling strength or feedback coefficient. Let the error vector be $\xi(t)=\mathbf{x}(t)-\mathbf{s}(t)$. According to (16), its variational equation is

$$
\dot{\xi}(t)=[D \mathbf{f}(\mathbf{s}(t))+d \mathbf{A}] \xi(t)
$$

Obviously, the associated feedback system (15) of network (1) is the response system in (18), and the individual node $\dot{\mathbf{x}}(t)=$ $\mathbf{f}(\mathbf{x}(t))$ is the drive system of (18). Moreover, the variational (19) [(or (17)] is the corresponding linear system of the associated feedback system (15). If the origin is an exponentially stable equilibrium of system (19), then the unidirectional coupled system (18) is synchronous. Therefore, the associated feedback system (15) and the individual node of network (1) have their precise meaning in terms of synchronous communication.

\section{B. Characterizing the Robustness of Synchronization}

The robustness of synchronization is a key characteristic quantity of complex dynamical networks. Networks with different topological structures are likely to have different degrees of robustness of synchronization. Moreover, in a network, different edges may have different degrees of robustness of synchronization. It has been demonstrated that a scale-free network is robust against random removal of nodes but is fragile to specific removal of the most highly connected nodes [9]. Therefore, it is very important to give a mathematical characterization for the robustness of synchronization. In the following, a new concept of synchronizability matrix is introduced to characterize the robustness of synchronization for dynamical network (1). 
Definition 4: Suppose that network (1) can achieve synchronization for a given inner coupled matrix $\mathbf{A}$ and a coupled configuration matrix $\mathbf{C}$. If network (1) remains synchronized after removing one edge, $l$, from the network, then network (1) is said to be robust in synchronization against the removal of edge $l$; otherwise, network (1) is said to be fragile in synchronization with respect to the removal of edge $l$.

Definition 5: Consider the dynamical network (1), and let $\Gamma$ be the exponentially stable region of the zero solution of system (17) with respect to feedback parameter $d$. The real matrix

$$
\mathbf{S}=\left(s_{i j}\right)_{N \times N}
$$

is called the synchronizability matrix for one-edge removal of network (1), where

$$
\begin{aligned}
s_{i j} & =\alpha\left(\lambda_{2}^{i j}, \lambda_{3}^{i j}, \ldots, \lambda_{N}^{i j}\right) \\
& = \begin{cases}0, & \text { if } j=i \text { or } c_{i j}=0 \\
\left\{\begin{array}{l}
1, \text { if } \lambda_{k}^{i j} \in \Gamma, \text { for } k=2, \ldots, N, \\
-1, \text { otherwise },
\end{array}\right. & \text { otherwise }\end{cases}
\end{aligned}
$$

in which $\lambda_{k}^{i j}(2 \leq k \leq N)$ are the nonzero eigenvalues of transformation matrix $\mathbf{C}^{i j}=\left(c_{l m}^{i j}\right)_{N \times N}$ satisfying the following: If $l=i$ and $m=j$, then $c_{i j}^{i J}=0$ and $c_{i i}^{i j}=c_{i i}+c_{i j}$; otherwise, $c_{l m}^{i j}=c_{l m}$. If $\mathbf{S} \geq \mathbf{0}$, then the dynamical network (1) is said to be globally robust in synchronization against one-edge removal; if $\mathbf{S} \leq \mathbf{0}$, then the dynamical network (1) is said to be globally fragile in synchronization with respect to one-edge removal. If $s_{i j}=1$, then, the edge from node $i$ to node $j$ is robust in synchronization against removal and this edge is called a robust edge of network (1); if $s_{i j}=-1$, then the edge from node $i$ to node $j$ is fragile in synchronization with respect to removal, and this edge is called a sensitive edge of network (1).

Note that the synchronizability matrix $\mathbf{S}$ characterizes the robustness of synchronization against one-edge removal of network (1). Moreover, the synchronizability matrix $\mathbf{S}$ concretizes the robustness and fragility of synchronization against one-edge removal of network (1). Similarly, one can introduce the synchronizability matrices for multi-edge removals of network (1).

For the case of adding edges, one can similarly define the synchronizability matrix of one-edge addition (or multi-edge addition) for network (1). The only difference is that in this case

$$
\begin{aligned}
s_{i j} & =\alpha\left(\lambda_{2}^{i j}, \lambda_{3}^{i j}, \ldots, \lambda_{N}^{i j}\right) \\
& = \begin{cases}0, & \text { if } j=i \text { or } c_{i j} \neq 0 \\
\left\{\begin{array}{l}
1, \text { if } \lambda_{k}^{i j} \in \Gamma \text { for } k=2, \ldots, N \\
-1, \text { otherwise }
\end{array}\right. & \text { otherwise }\end{cases}
\end{aligned}
$$

where $\lambda_{k}^{i j}(2 \leq k \leq N)$ are the nonzero eigenvalues of the transformation matrix $\mathbf{C}^{i j}=\left(c_{l m}^{i j}\right)_{N \times N}$ satisfying the following: If $l=i$ and $m=j$, then $c_{i j}^{i j}=c_{i j}^{\prime}$ and $c_{i i}^{i j}=c_{i i}-c_{i j}^{\prime}$; otherwise, $c_{l m}^{i j}=c_{l m}$.

Suppose that $\left(\mathbf{A}+\mathbf{A}^{T}\right)$ is a positive semi-definite matrix, and let $T=\left\{d \mid \mu[D \mathbf{f}(\mathbf{s}(t))+d \mathbf{A}] \leq a<0\right.$ for $\left.t \geq t_{0}\right\}$. According to the stability theory of linear time-varying systems [18], if $d \in T$, then, the linear time-varying system (17) is exponentially stable about its zero solution. Therefore, $T \subseteq \Gamma$. As- sume that $\lambda_{k}(1 \leq k \leq N)$ are real. From (3) and Lemma 2 (or Lemma 4), if $\lambda_{2} \in T$ (or $0 \in T$ ), then, the chaotic synchronous state $\mathbf{x}_{1}(t)=\mathbf{x}_{2}(t)=\cdots=\mathbf{x}_{N}(t)=\mathbf{s}(t)$ (or the synchronous solution $\left.\mathbf{S}(t)=\left(\mathbf{s}^{T}(t), \mathbf{s}^{T}(t), \ldots, \mathbf{s}^{T}(t)\right)^{T}\right)$ of dynamical network (1) is exponentially stable.

Suppose that $\mathbf{s}(t)$ is a chaotic orbit of the individual node $\dot{\mathbf{x}}(t)=\mathbf{f}(\mathbf{x}(t))$. Then $\dot{\mathbf{x}}(t)=D \mathbf{f}(\mathbf{s}(t))$ is unstable. That is, $0 \notin \Gamma$. Theoretical analysis and numerical simulations both show that $\Gamma=(-\infty, b](b<0)$ for most coupled matrices $\mathbf{A}=\operatorname{diag}\left\{a_{11}, \ldots, a_{n n}\right\} \geq 0$ [11]. Since $\dot{\mathbf{x}}(t)=D \mathbf{f}(\mathbf{s}(t))$ is not stable, in order to make system (17) exponentially stable, the feedback $d \mathbf{A} \mathbf{y}(t)$ should be a negative feedback. Since $\mathbf{A}=\operatorname{diag}\left\{a_{11}, \ldots, a_{n n}\right\} \geq 0$, one has $d<0$. Assume that $\lambda_{i}(1 \leq i \leq N)$ are real. From Theorem 1 and (3), the chaotic synchronous state $\mathbf{x}_{1}(t)=\mathbf{x}_{2}(t)=\cdots=\mathbf{x}_{N}(t)=\mathbf{s}(t)$ of dynamical network (1) is exponentially stable if and only if $\lambda_{2} \leq b$. That is, the exponential stability of synchronous state $\mathbf{x}_{1}(t)=\mathbf{x}_{2}(t)=\cdots=\mathbf{x}_{N}(t)=\mathbf{s}(t)$ of network (1) is completely determined by the exponential stability of the linear time-varying system

$$
\dot{\mathbf{y}}(t)=\left[D \mathbf{f}(\mathbf{s}(t))+\lambda_{2} \mathbf{A}\right] \mathbf{y}(t) .
$$

Therefore, linear time-varying system (23) determines the synchronizability of network (1).

Let $d_{\max }$ be the maximum of the feedback parameter $d$, which can exponentially stabilize the associated feedback system (15) about $\mathbf{x}(t)=\mathbf{s}(t)$. Obviously, $d_{\max }=b$. Then, (21) can be simplified as follows:

$$
\begin{aligned}
s_{i j} & =\alpha\left(\lambda_{2}^{i j}\right) \\
& = \begin{cases}0, & \text { if } j=i \text { or } c_{i j}=0 \\
\left\{\begin{array}{l}
1, \text { if } \lambda_{2}^{i j} \leq d_{\max } \\
-1, \text { otherwise }
\end{array}\right. & \text { otherwise } .\end{cases}
\end{aligned}
$$

Thus, the maximum nonnegative eigenvalue (or the second-largest eigenvalue) $\lambda_{2}$ of the coupling configuration matrix $\mathbf{C}$ determines the synchronizability of the dynamical network (1): if $\lambda_{2}(\mathbf{C}) \leq d_{\max }(\mathbf{f}(\mathbf{s}(t)), \mathbf{A})$, then network (1) will synchronize; otherwise, it will not synchronize. Therefore, $d_{\max }$ is the synchronous threshold of the dynamical network (1). This is in sharp contrast to the case where $\mathbf{s}(t)$ is chaotic.

If $\mathbf{s}(t)$ is an exponentially stable solution of $\dot{\mathbf{x}}(t)=\mathbf{f}(\mathbf{x}(t))$, then $0 \in \Gamma$. Here, in order to make system (17) exponentially stable, the feedback $d \mathbf{A y}(t)$ need not be a negative feedback.

\section{Comparing With Regular Coupled Networks}

Section II has reviewed two typical small-world network models: the WS model and the NW model. It is noticed that the NW model reduces to the nearest-neighbor coupling network for $p=0$, while it becomes a globally coupling network for $p=1$. Obviously, the small-world network bridges the gap between the nearest-neighbor coupling network and the globally coupling network and realizes the transformation from one to the other. It has been shown that for any given coupling strength, if the number of nodes is large enough, then the globally coupling network eventually synchronizes, while the nearest-neighbor coupling network cannot achieve synchronization under the same condition. Recently, Wang and 


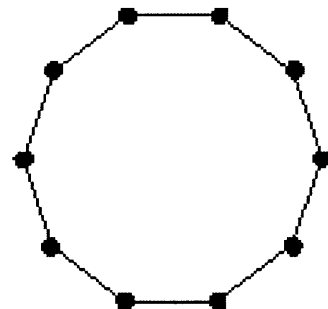

[a]

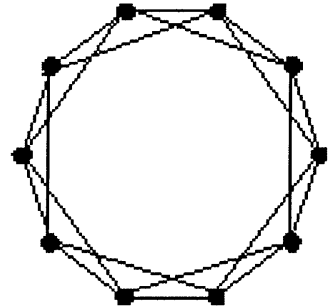

[(b)

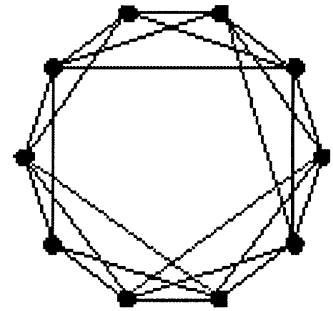

[c]

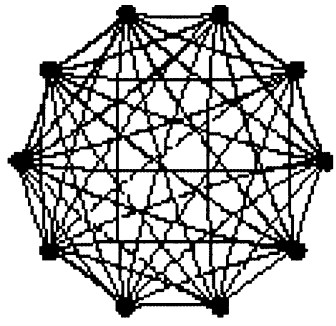

[d]

Fig. 2. Network (1) with ten nodes. (a) Simplest nearest-neighbor coupling network. (b) Nearest-neighbor coupling network with four nearest-neighbor coupled nodes. (c) Small-world network. (d) Fully connected network.

Chen [8] show that, for any given coupling strength, and with a sufficiently large number of nodes, a uniform small-world dynamical network will synchronize. In the following, it will explain why they have different situations of synchronizability by analyzing their synchronizability matrices for the case where $\mathbf{s}(t)$ is a chaotic orbit of the individual node $\dot{\mathbf{x}}(t)=\mathbf{f}(\mathbf{x}(t))$.

The nearest-neighbor coupling network consists of nodes being arranged in a ring and coupled to the nearest neighbors. Fig. 2(a) shows a single nearest-neighbor coupling network. Assume that $\mathbf{A}=\operatorname{diag}\{1,0, \ldots, 0\}$ and the corresponding coupling configuration matrix is

$$
\mathbf{C}_{1}=\left(\begin{array}{ccccc}
-2 & 1 & & & 1 \\
1 & -2 & 1 & & \\
& \ddots & \ddots & \ddots & \\
& & 1 & -2 & 1 \\
1 & & & 1 & -2
\end{array}\right)
$$

Its eigenvalues are

$$
\left\{-4 \sin ^{2}\left(\frac{k \pi}{N}\right), k=0,1, \ldots, N-1\right\}
$$

Assume that $\Gamma=\left(-\infty, d_{\max }\right]\left(d_{\max }<0\right)$. If

$$
d_{\max } \geq-4 \sin ^{2}\left(\frac{\pi}{N}\right)
$$

then, the nearest-neighbor coupling network will asymptotically synchronize.

For $0<p<1$, the NW model is a small-world network and can be described by the dynamical network model (1). Moreover, the small-world network can be generated as follows: in the nearest-neighbor coupling network $\mathbf{C}_{1}$, if $c_{i j}=0$, then let $c_{i j}=c_{j i}=1$ with probability $p$. Thus, one obtains the coupling configuration matrix $\mathbf{C}$ and its second-largest eigenvalue $\lambda_{2}$. It is known that if

$$
d_{\max } \geq \lambda_{2}
$$

then the small-world network will synchronize. Numerical results show that $\lambda_{2}$ will asymptotically decrease toward $-N$ as $p \rightarrow 1$ for any given $N$. Moreover, there exists a critical value $\hat{p}$ such that the small-world network (1) will synchronize for $\hat{p} \leq p \leq 1$. These results coincide with the numerical results [8, Fig. 1] and the theoretical analysis of [8]. For a given probability $p$, if one calculates its corresponding synchronizability matrix $\mathbf{S}$ for one-edge addition, then it can be seen that the synchronizability of the small-world network (1) increases. Also, there exists a critical value $\bar{p}$ satisfying $\mathbf{S} \geq 0$ for $\bar{p} \leq p \leq 1$, which means that the small-world network (1) is synchronous and is also robust against one-edge addition for $\bar{p} \leq p \leq 1$. Furthermore, $\hat{p} \approx \bar{p}$. Therefore, these theoretical results coincide with the numerical results of [8]. Here, the critical probability $\hat{p}$ (or $\bar{p}$ ) is called the saturated synchronization probability.

For $p=1$, the NW model becomes a globally coupled network, as shown in Fig. 2(d). The corresponding coupling configuration matrix is

$$
\mathbf{C}_{2}=\left(\begin{array}{ccccc}
-N+1 & 1 & 1 & \cdots & 1 \\
1 & -N+1 & 1 & \cdots & 1 \\
\vdots & \ddots & \ddots & \ddots & \vdots \\
1 & 1 & 1 & \cdots & 1 \\
1 & 1 & 1 & \cdots & -N+1
\end{array}\right)
$$

and its eigenvalues are 0 with multiplicity 1 and $-N$ with multiplicity $N-1$. Similarly, if

$$
d_{\max } \geq-N
$$

then, the globally coupled network will synchronize, which means that, for any given irreducible coupling configuration matrix $\mathbf{C} \geq 0$, the globally coupled network will synchronize when the number of nodes is large enough. Note that $d_{\max }$ is only dependent on the individual node $\dot{\mathbf{x}}(t)=\mathbf{f}(\mathbf{x}(t))$ and the coupling matrix $\mathbf{A}$, which implies that the irreducible coupling configuration matrix $\mathbf{C}$ may be arbitrarily small for a given $N$ satisfying (30). However, for a given large $N$, (27) shows that the nearest-neighbor coupling network will not synchronize for any large coupling configuration matrix $\mathbf{C}$.

In the above, a large enough $N$ is chosen to satisfy (30) but not (27). That is, for a given large $N$, the nearest-neighbor coupling network may not synchronize but the globally coupled network does. Therefore, in the NW model, the synchronizability of the dynamical network (1) will gradually increase when the probability $p$ increases from 0 to 1 .

\section{SEVERAL EXAMPLES}

\section{A. The Example of Simulation}

In the following, Theorem 1 and Definition 5 are illustrated by using the Lorenz system as the dynamical node in network (1). For simplicity, consider network (1) with ten nodes as 


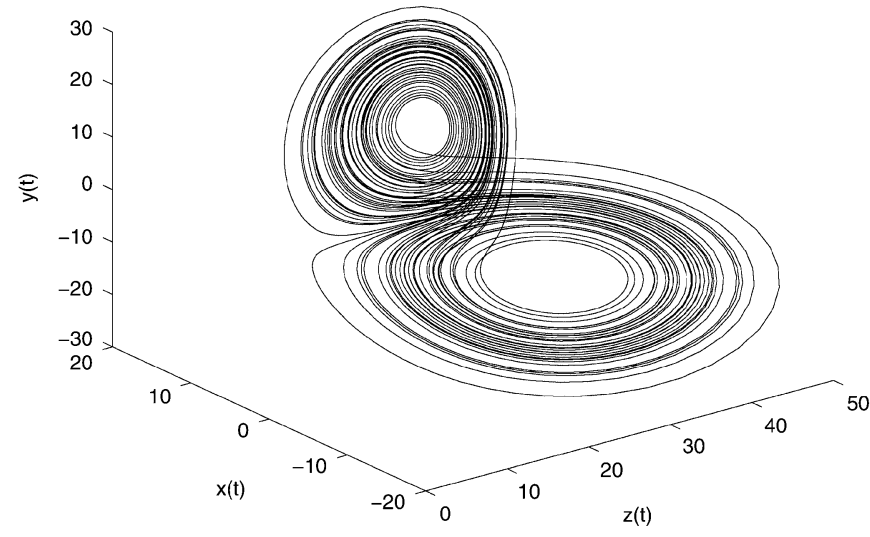

Fig. 3. Lorenz chaotic attractor. ( $a=10, b=8 / 3, c=28)$.

shown in Fig. 2. The classical Lorenz system of node $i$ is described by [13]

$$
\left(\begin{array}{c}
\dot{x}_{i 1} \\
\dot{x}_{i 2} \\
\dot{x}_{i 3}
\end{array}\right)=\left(\begin{array}{c}
a\left(x_{i 2}-x_{i 1}\right) \\
c x_{i 1}-x_{i 1} x_{i 3}-x_{i 2} \\
x_{i 1} x_{i 2}-b x_{i 3}
\end{array}\right), \quad i=1, \ldots, 10
$$

which has a chaotic attractor as displayed in Fig. 3 when $a=$ $10, b=8 / 3, c=28$. The system Jacobian is

$$
D \mathbf{f}\left(x_{i}\right)=\left(\begin{array}{ccc}
-a & a & 0 \\
c-x_{i 3} & -1 & -x_{i 1} \\
x_{i 2} & x_{i 1} & -b
\end{array}\right)
$$

Fig. 2 shows four typical network structures. Their average path lengths $L$ and clustering coefficients $C$ are, respectively, as follows:

- Fig. 2(a), a nearest-neighbor coupling network: $L_{\mathrm{nnc}}=$ $25 / 9$ and $C_{\mathrm{nnc}}=0$;

- Fig. 2(b), a nearest-neighbor coupling network: $L_{2 \mathrm{nnc}}=$ $5 / 3$ and $C_{2 \mathrm{nnc}}=1 / 2$;

- Fig. 2(c), a small-world network: $L_{\mathrm{sw}}=22 / 15$ and $C_{\mathrm{sw}}=89 / 150$

- Fig. 2(d), a fully connected network: $L_{\mathrm{fc}}=1$ and $C_{\mathrm{fc}}=1$.

In the following, four typical network structures are studied, as shown in Fig. 2 with different inner coupled matrix $\mathbf{A}: \mathbf{A}_{1}=$ $\operatorname{diag}\{1,0,0\}, \mathbf{A}_{2}=\operatorname{diag}\{0,1,0\}, \mathbf{A}_{3}=\operatorname{diag}\{0,0,1\}, \mathbf{A}_{4}=$ $\operatorname{diag}\{1,1,1\}$. From (15), the associated feedback system of network (1) is

$$
\left(\begin{array}{c}
\dot{x}_{11} \\
\dot{x}_{12} \\
\dot{x}_{13}
\end{array}\right)=\left(\begin{array}{c}
a\left(x_{12}-x_{11}\right) \\
c x_{11}-x_{11} x_{13}-x_{12} \\
x_{11} x_{12}-b x_{13}
\end{array}\right)+d \mathbf{A}\left(\begin{array}{l}
x_{11}-s_{1}(t) \\
x_{12}-s_{2}(t) \\
x_{13}-s_{3}(t)
\end{array}\right)
$$

where $\mathbf{s}(t)=\left(s_{1}(t), s_{2}(t), s_{3}(t)\right)^{T}$ is an orbit of the Lorenz chaotic attractor. Numerical results show that the exponentially stable regions of the zero solution of system (33) in terms of feedback parameter $d$ are

$$
\begin{array}{ll}
\Gamma_{1}=(-\infty,-7.90], & \text { for } \mathbf{A}_{1}=\operatorname{diag}\{1,0,0\} \\
\Gamma_{2}=(-\infty,-2.77], & \text { for } \mathbf{A}_{2}=\operatorname{diag}\{0,1,0\} \\
\Gamma_{3}=[-6.30,-1.46], & \text { for } \mathbf{A}_{3}=\operatorname{diag}\{0,0,1\} \\
\Gamma_{4}=(-\infty,-0.92], & \text { for } \mathbf{A}_{4}=\operatorname{diag}\{1,1,1\} .
\end{array}
$$

According to Theorem 1, the chaotic synchronous state $\mathbf{x}_{1}(t)=$ $\mathbf{x}_{2}(t)=\cdots=\mathbf{x}_{10}(t)=\mathbf{s}(t)$ of dynamical network (1) is exponentially stable if and only if the following conditions hold:

$\lambda_{2} \leq-7.90, \quad$ for $\mathbf{A}_{1}=\operatorname{diag}\{1,0,0\}$

$\lambda_{2} \leq-2.77, \quad$ for $\mathbf{A}_{2}=\operatorname{diag}\{0,1,0\}$

$\lambda_{2} \leq-1.46$ and $\lambda_{10}(t) \geq-6.30, \quad$ for $\mathbf{A}_{3}=\operatorname{diag}\{0,0,1\}$

$\lambda_{2} \leq-0.92, \quad$ for $\mathbf{A}_{4}=\operatorname{diag}\{1,1,1\}$.

Let $\mathbf{A}=\mathbf{A}_{2}=\operatorname{diag}\{1,1,1\}$. Only calculate the synchronizability matrix for one-edge removal of the small-world network [Fig. 2(c)]. The coupled configuration matrix is shown in (36) at the bottom of the page, and its synchronizability matrix (for one-edge removal) is

$$
\mathbf{S}=\left(\begin{array}{llllllllll}
0 & 1 & 1 & 0 & 0 & 0 & 0 & 0 & 1 & 1 \\
1 & 0 & 1 & 1 & 0 & 0 & 0 & 0 & 1 & 1 \\
1 & 1 & 0 & 1 & 1 & 1 & 0 & 0 & 0 & 0 \\
0 & 1 & 1 & 0 & 1 & 1 & 0 & 0 & 0 & 0 \\
0 & 0 & 1 & 1 & 0 & 1 & 1 & 1 & 0 & 0 \\
0 & 0 & 1 & 1 & 1 & 0 & 1 & 1 & 0 & 0 \\
0 & 0 & 0 & 0 & 1 & 1 & 0 & 1 & 1 & 1 \\
0 & 0 & 0 & 0 & 1 & 1 & 1 & 0 & 1 & 1 \\
1 & 1 & 0 & 0 & 0 & 0 & 1 & 1 & 0 & 1 \\
1 & 1 & 0 & 0 & 0 & 0 & 1 & 1 & 1 & 0
\end{array}\right)
$$

Since $\lambda_{2}=-2.2907<-0.92$, the small-world network will synchronize. Moreover, $\mathbf{S} \geq 0$, so the small-world network ex-

$$
\mathbf{C}=\left(\begin{array}{cccccccccc}
-4 & 1 & 1 & 0 & 0 & 0 & 0 & 0 & 1 & 1 \\
1 & -5 & 1 & 1 & 0 & 0 & 0 & 0 & 1 & 1 \\
1 & 1 & -5 & 1 & 1 & 1 & 0 & 0 & 0 & 0 \\
0 & 1 & 1 & -4 & 1 & 1 & 0 & 0 & 0 & 0 \\
0 & 0 & 1 & 1 & -5 & 1 & 1 & 1 & 0 & 0 \\
0 & 0 & 1 & 1 & 1 & -5 & 1 & 1 & 0 & 0 \\
0 & 0 & 0 & 0 & 1 & 1 & -5 & 1 & 1 & 1 \\
0 & 0 & 0 & 0 & 1 & 1 & 1 & -5 & 1 & 1 \\
1 & 1 & 0 & 0 & 0 & 0 & 1 & 1 & -5 & 1 \\
1 & 1 & 0 & 0 & 0 & 0 & 1 & 1 & 1 & -5
\end{array}\right)
$$


hibits global robustness of synchronization against one-edge removal, and all edges are robust edges.

\section{B. Some Examples of Applications}

As seen from the above, the synchronizability matrix $\mathbf{S}$ is a powerful tool for analyzing the robustness of synchronization for network (1). In fact, one can improve or construct some small-world dynamical network models for possible engineering applications based on the knowledge of synchronizability.

For example, consider a small-world network model of common vibration. For a given nearest-neighbor coupling network, how can one add some edges (i.e., links) to make the whole network simultaneously vibrate (i.e., synchronize)? It is noticed that the more edges, the more difficult to implement. Let $r$ be the number of the edges added that can then make the network synchronize. Assume that the number of nodes $N$ is large enough to make the corresponding globally coupled network synchronize. Now, compare the results of two different ways of adding edges: in one way, the edges are added at random and in the other they are chosen to be robust edges based on the knowledge of the synchronizability matrix. It is clear that the choice of edges using robust edges has a definite advantage over the random choice. That is, the number of robust edges is clearly less than that of random additions.

Similar methods can be applied to real-world communication networks. In many communication networks, synchronous transfers of digital or analog signals are very important. Sometimes, one needs to construct or improve the existing network to increase its synchronizability. In doing so, one only needs to add or adjust several edges using the knowledge of synchronizability.

Nowadays, the robustness of synchronization of complex networks has become more and more important for network security. For a large-scale complex dynamical network, the random failures and attacks to the network structure or connections are often unavoidable, for example, the recent King of Worm, on 25 January 2003 spreading over the Internet. In some real smallworld networks, there are some sensitive edges that are easily attacked. These key edges should be replaced by adding some robust edges so as to increase the robustness of synchronization of the network.

It is shown that the spread of an epidemic (or computer virus) is much faster in small-world networks than in regular networks, and the speed is almost close to that in random networks. How does an epidemic (or a computer virus) spread on the social network (or the Internet)? Recently, many researchers have already obtained some elementary results that address this issue [29], [30]. It is now possible to control the spread of a computer virus or increase the advertisement function of a network by using the concepts of sensitive and robust edges.

Theoretical analysis and numerical simulations show that there are many similarities and differences regarding the synchronizability of dynamical systems on small-world and scale-free networks. In general, the small-world networks are easier to realize network synchronization [8]. However, the scale-free networks are more robust against random removal of nodes, but is more fragile to specific removal of the most highly clustering nodes [9].

\section{CONCLUSION}

In this paper, it has been shown that maximum synchronizability of a network is completely determined by its associated feedback system. Also, a new concept-the synchronizability matrix - can be used to characterize the maximum synchronizability of the network. Moreover, the concepts of sensitive edge and robust edge are useful for analyzing the robustness and fragility of synchronization of the network. Based on the knowledge of synchronizability, one can purposefully increase the robustness of synchronization thereby protecting against attacks to the small-world networks.

The sensitive edges of a small-world network have a higher degree of influence on the network synchronization, which is very important for increasing or controlling the synchronizability of the network. The mechanism of sensitive edges need to be further explored in the near future. Our results strongly indicate that for a small-world dynamical network, the associated feedback system, the synchronizability matrix, and robust and sensitive edges are the key elements responsible for the special network properties, such as the maximum synchronizability and the robustness and fragility of synchronization. However, how to construct a robust small-world network for network security, say protecting against attacks to the Internet, remains an important but challenging problem.

\section{REFERENCES}

[1] M. E. J. Newman, "Models of the small world: A Review," J. Stat. Phys., vol. 101, pp. 819-841, 2000.

[2] M. Marchiori and V. Latora, "Harmony in the small-world," Physica A, vol. 285, pp. 539-546, 2000.

[3] S. A. Pandit and R. E. Amritkar, "Characterization and control of smallworld networks," Phys. Rev. E, vol. 60, no. 2, pp. 1119-1122, 1999.

[4] D. J. Watts and S. H. Strogatz, "Collective dynamics of small-world," Nature, vol. 393, pp. 440-442, 1998.

[5] M. E. J. Newman and D. J. Watts, "Scaling and percolation in the smallworld network model," Phys. Rev. E, vol. 60, no. 6, pp. 7332-7342, 1999.

[6] T. Nishikawa, A. E. Motter, Y.-C. Lai, and F. C. Hoppensteadt, "Smallest small-world network," Phys. Rev. E, vol. 66, no. 4, 046 139, 2002.

[7] V. J. Yung, An Exploration in the Small-World Networks, Singapore: Faculty of Business Administration, National Univ. Singapore, 2001.

[8] X. Wang and G. Chen, "Synchronization in small-world dynamical networks," Int. J. Bifurcation Chaos, vol. 12, no. 1, pp. 187-192, 2002.

[9] —-, "Synchronization in scale-free dynamical networks: Robustness and fragility," IEEE Trans. Circuits Syst. I, vol. 49, pp. 54-62, Jan. 2002.

[10] J. Lü, X. Yu, and G. Chen, "Chaos synchronization of general complex dynamical networks," Phys. A, vol. 334, pp. 281-302, 2004.

[11] J. Lü, T. Zhou, and S. Zhang, "Chaos synchronization between linearly coupled chaotic system," Chaos Solitons Fractals, vol. 14, no. 4, pp. 529-541, 2002.

[12] J. Lü, J. Lu, and S. Chen, Chaotic Time Series Analysis and Its Applications. Wuhan, China: Wuhan Univ. Press, 2002.

[13] G. Chen and X. Dong, From Chaos to Order: Methodologies, Perspectives, and Applications, Singapore: World Scientific, 1998.

[14] K. Kaneko, Ed., Coupled Map Lattices., Singapore: World Scientific, 1992.

[15] S. C. Manrubia and S. M. Mikhailov, "Mutual synchronization and clustering in randomly coupled chaotic dynamical networks," Phys. Rev. E, vol. 60, no. 2, pp. 1579-1589, 1999.

[16] C. W. Wu, "Synchronization in an array of linearly coupled dynamical systems," IEEE Trans. Circuits Syst. I, vol. 42, pp. 430-447, Aug. 1995. 
[17] M. Barthélémy and L. A. N. Amaral, "Small-world networks: Evidence for a cross over picture," Phys. Rev. Lett., vol. 82, no. 15, pp. 3180-3183, 1999.

[18] M. E. J. Newman and D. J. Watts, "Renormalization group analysis of the small-world network model," Phys. Lett. A, vol. 263, pp. 341-346, 1999.

[19] R. V. Kulkarni, E. Almaas, and D. Stroud, "Exact results and scaling properties of small-world networks," Phys. Rev. E, vol. 61, no. 4, pp. $4268-4271,2000$.

[20] M. E. J. Newman, C. Moore, and D. J. Watts, "Mean-field solution of the small-world network model," Phys. Rev. Lett., vol. 84, no. 14, pp. 3201-3204, 2000.

[21] E. Almaas, R. V. Kulkarni, and D. Stoud, "Characterizing the structure of small-world networks," Phys. Rev. Lett., vol. 88, no. 9, 098 101, 2002.

[22] R. Albert and A.-L. Barabási, "Statistical mechanics of complex networks," Rev. Mod. Phys., vol. 74, pp. 47-97, 2002.

[23] M. Barahona and L. M. Pecora, "Synchronization in small-world systems," Phys. Rev. Lett., vol. 89, no. 5, 054 101, 2002.

[24] H. Hong, M. Y. Choi, and B. J. Kim, "Synchronization on small-world networks," Phys. Rev. E, vol. 65, no. 5, 026139, 2002

[25] L. Cisneros, J. Jimenez, M. G. Cosenza, and A. Parravano, "Information transfer and nontrivial collective behavior in chaotic coupled map networks," Phys. Rev. E, vol. 65, no. 4, 045 204, 2002.

[26] J. Jost and M. P. Joy, "Spectral properties and synchronization in coupled map lattices," Phys. Rev. E, vol. 65, no. 1, 016201, 2002.

[27] R. Albert, H. Jeong, and A.-L. Barabási, "Error and attack tolerance of complex networks," Nature, vol. 406, pp. 378-382, 2000.

[28] H. K. Khalil, Nonlinear Systems. Englewood Cliffs, NJ: Prentice-Hall, 1996.

[29] D. J. Watts, "A simple model of fads and cascading failures," Santa Fe Institute Working Paper, 2000.

[30] P. Svenson and D. A. Johnston, Damage Spreading in Small in Small World Ising Models. Ithaca, NY: Cornell Univ., 2001.

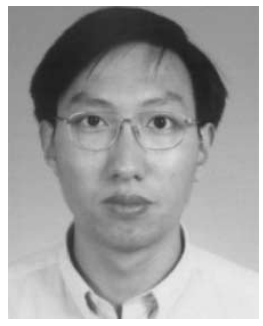

Jinhu Lü (M'03) was born in China in 1974. He received the B.Sc. degree in mathematics, the M.Sc. degree in applied mathematics, and $\mathrm{Ph} . \mathrm{D}$. degree in applied mathematics, from Hubei Normal University, Hubei, China, Wuhan University, Wuhan, China, and the Chinese Academy of Sciences, Beijing, China, in 1997, 2000, and 2002, respectively.

From January to April of 2002, he was a Research Assistant, and from August to November of 2003, a Senior Research Associate at the Centre for Chaos Control and Synchronization, City University of Hong Kong, Hong Kong. He was a Visiting Research Fellow in the School of Electrical and Computer Engineering, Royal Melbourne Institute of Technology University, Melbourne, Australia, from January to May 2003. From June 2002 to March 2004, he was a Postdoctoral Research Fellow and is currently an Assistant Professor with the Institute of Systems Science, Chinese Academy of Sciences, Beijing, China. He is the author of two research monographs and more than 40 research journal papers published in the fields of control and synchronization of complex dynamical systems.

Dr. Lü received the Presidential Outstanding Research Award from the Chinese Academy of Sciences in 2002.

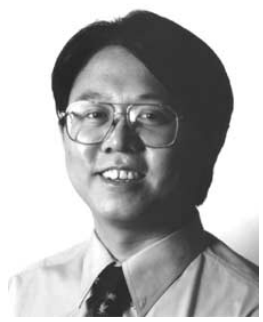

Xinghuo Yu (M'91-SM'96) received the B.E. and M.E. degrees from the University of Science and Technology, Hefei, China, in 1982 and 1985, respectively, and the Ph.D. degree from South-East University, Nanjing, China, in 1988.

From 1987 to 1989 , he was a Research Fellow with the Institute of Automation, Chinese Academy of Sciences, Beijing, China. From 1989 to 1991, he was a Postdoctoral Fellow in the Applied Mathematics Department, University of Adelaide, Adelaide, Australia. From 1991 to 2002, he was with Central Queensland University, Queensland, Australia where, before he left in
March 2002, he was Professor and the Associate Dean (Research) of the Faculty of Informatics and Communication. Since March 2002, he has been with the RMIT University, Melbourne, Australia, where he is now a Professor and the Associate Dean (Research and Development) of the Faculty of Engineering. He has also held Visiting Professor positions at the City University of Hong Kong, Hong Kong, and the Bogazici University, Ankara, Turkey. He is a Guest Professor of Harbin Institute of Technology, Harbin, China, the Huazhong University of Science and Technology,Wuhan, China, and Southeast University, Nanjing, China. His research interests include sliding-mode and nonlinear control, chaos and chaos control, and soft computing and applications. He has published over 200 refereed papers in technical journals, books, and conference proceedings. He has also coedited seven research books.

Prof. Yu has served as an Associate Editor of IEEE TRANSACTIONS ON CIRCUITS AND SYSTEMS-I between 2001 and 2003. He has been on program committees of many international conferences, and has chaired/co-chaired Complex 1994, VSS 2000, Complex 2004 and AI 2004. He was the sole recipient of the 1995 Central Queensland University Vice Chancellor's Award for Research. He is a Fellow of the Institution of Engineers Australia. He was conferred Emeritus Professor of Central Queensland University in 2002.

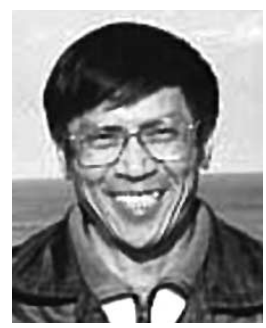

Guanrong Chen (M'87-SM'92-F'96) received the M.Sc. degree in computer science from the Sun Yatsen (Zhongshan) University, Guangzhou, China, and the Ph.D. degree in applied mathematics from Texas A\&M University, College Station.

Currently, he is a Chair Professor at the City University of Hong Kong, Hong Kong, and the Director of the Centre for Chaos Control and Synchronization therein. He has numerous publications since 1981, in the fields of nonlinear systems, in both dynamics and controls, which include 15 monographs and textbooks, 280 journal papers, and 180 conference abstracts. Among his publications are the research monographs entitled Hopf Bifurcation Analysis (Singapore: World Scientific, 1996), From Chaos to Order (Singapore: World Scientific, 1998), and edited books Controlling Chaos and Bifurcations in Engineering Systems (Boca Raton: FL, CRC, 1999), Chaos in Circuits and Systems (Singapore: World Scientific, 2002), Chaos Control, and Bifurcation Control (New York: Springer-Verlag, 2003).

Prof. Chen served and is serving as Editor in various capacities for eight international journals, including the IEEE TRANSACTIONS ON CIRCUITS AND Systems, the IEEE MAGAZINE ON CIRCUITS AND SySTEMS, and the International Journal of Bifurcation and Chaos. He received the 1998 Harden-Simons Prize for the Outstanding Journal Paper Award from the American Society of Engineering Education, the $2001 \mathrm{M}$. Barry Carlton Best Transactions Paper Award from the IEEE Aerospace and Electronic Systems Society, and the 2002 Best Paper Award from the Institute of Information Theory and Automation, Academy of Sciences of the Czech Republic. He is an Honorary Professor of the Central Queensland University, Australia, as well as Honorary Guest-Chair Professor of several universities in China.

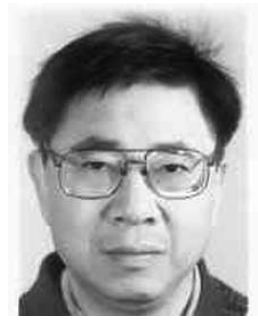

Daizhan Cheng (SM'01) received the Ph.D. degree from Washington University, St. Louis, MO, in 1985.

Since 1990, he has been a Professor with the Institute of Systems Science, Chinese Academy of Sciences, Beijing, China. His research interests include nonlinear system and control, and numerical methods.

He was an Associate Editor of Mathematical Systems and Control from 1991-1993 and Automatica from 1998 to 2002. He is currently an Associate Editor of the Asian Journal of Control, Deputy Chief Editor of Control and Decision, and the Journal of Control Theory and Applications. He is the Chairman of the Technical Committee on Control Theory, and the Chinese Automation Association. 